\title{
Light cone and Weyl compatibility of conformal and projective structures
}

\author{
Vladimir S. Matveev ${ }^{1} \cdot$ Erhard Scholz $^{2}$ (D)
}

Received: 28 January 2020 / Accepted: 18 June 2020 / Published online: 3 July 2020

(c) The Author(s) 2020

\begin{abstract}
In the literature different concepts of compatibility between a projective structure $\mathscr{P}$ and a conformal structure $\mathscr{C}$ on a differentiable manifold are used. In particular compatibility in the sense of Weyl geometry is slightly more general than compatibility in the Riemannian sense. In an often cited paper (Ehlers et al. in: O'Raifertaigh (ed) General Relativity, Papers in Honour of J.L. Synge, Clarendon Press, Oxford, 2012) Ehlers/Pirani/Schild introduce still another criterion which is natural from the physical point of view: every light like geodesics of $\mathscr{C}$ is a geodesics of $\mathscr{P}$. Their claim that this type of compatibility is sufficient for introducing a Weylian metric has recently been questioned (Trautman in Gen Relativ Gravit 44:1581-1586, 2012); (Vladimir in Commun Math Phys 329:821-825, 2014); as reported by Scholz (in: A scalar field inducing a non-metrical contribution to gravitational acceleration and a compatible add-on to light deflection, 2019). Here it is proved that the conjecture of EPS is correct.
\end{abstract}

Keywords EPS compatibility $\cdot$ Weyl geometric compatibility $\cdot$ Foundations of gravity $\cdot$ Conformal structure $\cdot$ Projective structure

\section{Contents}

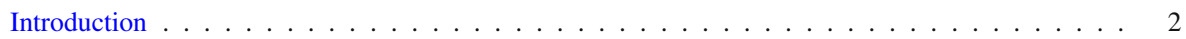

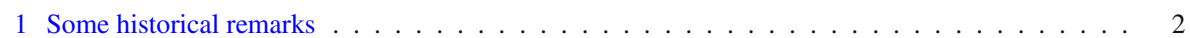

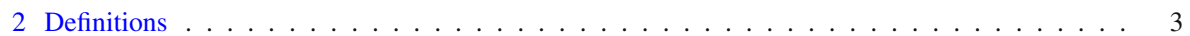

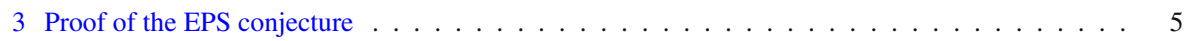

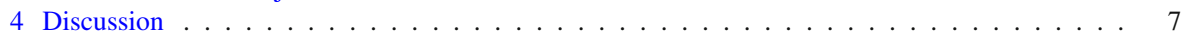

Erhard Scholz

scholz@math.uni-wuppertal.de

Vladimir S. Matveev

vladimir.matveev@uni-jena.de

1 Mathematics Institute, Faculty of Mathematics and Informatics, Friedrich-Schiller-Universität Jena, 07737 Jena, Germany

2 Faculty of Math./Natural Sciences, and Interdisciplinary Centre for Hist. and Phil. of Science, University of Wuppertal, Wuppertal, Germany 


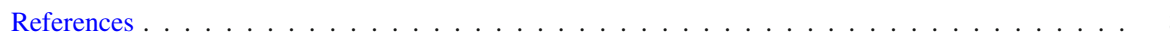

\section{Introduction}

In a widely read paper [7] J. Ehlers, F. Pirani and A. Schild (EPS) argued that a projective structure $\mathscr{P}$ and a conformal structure $\mathscr{C}$ on a differentiable manifold $M$ determine a Weylian metric on $M$, if only the geodesics of $\mathscr{P}$ are light like geodesics of $\mathscr{C}$. This statement complements a proposal of H. Weyl for basing the geometrical framework of gravity theory on the observable structures of particle trajectories and light propagation, rather than on the chronogeometric behaviour of clocks or rods [22]. EPS claimed that the above mentioned light-cone condition for $\mathscr{P}$ and $\mathscr{C}$ is sufficient for securing the existence of a Weylian metric which Weyl had assumed from the outset; but the argument given to substantiate the statement remains vague and is far from a mathematical proof [19]. The aim of the present paper is to fill in the gap and to prove the central statement of EPS.

The paper is structured as follows. We start with short remarks on the history of the problem (Sect. 1). After clarifying the central concepts involved in the question (Sect. 2) we prove the EPS conjecture (Sect. 3). Finally we discuss why we think it matters (Sect. 4).

\section{Some historical remarks}

In $1918 \mathrm{H}$. Weyl generalized the concept of a Riemannian metric in order to avoid the possibility of direct metrical comparison of vectors or other fields at finitely distant points $[20,21]$. He introduced a scale gauge connection in addition to a conformal structure, thus defining what later would be called a Weylian metric on a differentiable manifold (see below, Definition 1(ii)). In his book Space-Time-Matter and lectures given at Barcelona in 1922 he argued that the geometry of special relativity, i.e. the affine structure of Minkowski space, can be established without rods and clocks by projective geometry and the specification of a quadric at infinity in the sense of Klein's Erlangen program $[23,24]$. Generalizing this idea he argued that the geometrical structure of the general theory of relativity (GTR) can be based on the mathematical description of the inertial motion of test particles and light rays, rather than on the behaviour of rods and clock. He was able to underpin this view by showing that the generalized metrical structure of gravity, which he had proposed, i.e. a Weylian metric on $M$, is uniquely determined by its associated projective and and conformal structures [22]. ${ }^{1}$ He did not discuss, however, the conditions under which a projective structure and a conformal structure determine a Weylian metric.

About half a century later, in 1972, J. Ehlers, F. Pirani and A. Schild, sketched an even more ambitious program for establishing the fundamental conceptual framework of general relativity. They wanted to base even the differentiable structure on the set of spacetime events on more general, physically more or less plausible, assumptions

\footnotetext{
${ }^{1}$ See also Weyl's discussion with Einstein in 1918 [8, vol. 8B].
} 
(called "axioms") on the relation between events, particle paths and light propagation in spacetime [7]. This was considered as an attempt for a physically motivated "constructive axiomatics" of GTR and included the central claim that a projective structure and a conformal structure which are compatible on the light cones (see below Definition 2(i)) determine a Weylian metric on the spacetime manifold. ${ }^{2}$ The EPS paper led to a series of follow up investigations which in many cases concentrated on conditions which would reduce the Weyl geometric structure to a Riemannian (Lorentzian) one, often introducing additional information of quantum physics (Dirac field, complex scalar field) [1-5]. ${ }^{3}$ In these investigations the arguments of EPS were usually accepted, although the authors had qualified their arguments as not necessarily mathematically satisfying. ${ }^{4}$

The EPS paper was republished in 2012 as a by then classical text ("Golden Oldie") with an editorial comment by A. Trautman [19]. In his comments the editor raised doubts with regard to the status of the existence statement of EPS for a Weylian metric. He made clear that the arguments given in the original paper were rather vague and far from a mathematical proof. The existence statement ought thus to be considered a conjecture rather than a theorem as which it had been treated in large parts of the literature up to then. A first investigation of the case in a joint paper of Trautman with one of the present authors draws the conclusion that the EPS statement is wrong [12]. This judgment is based, however, on the criterion of Riemann compatibility between projective and conformal structures and thus on a too narrow understanding of the Weyl geometric setting. ${ }^{5}$ In the following argument it will be shown that the EPS conjecture is, in fact, true.

\section{Definitions}

We consider smooth manifolds and maps of class at least $\mathscr{C}^{2}$. All geometric objects on an $n \geq 3$-dimensional manifold are referred to local coordinates $\left(x^{i}\right), i=1, \ldots, n$.

A conformal structure on a manifold $M$ is an equivalence class $\mathscr{C}$ of metric tensors $g$ with respect to the following equivalence relation

$$
g \sim g^{\prime} \Longleftrightarrow \text { there is a function } \varphi \text { on } M \text { such that } g^{\prime}=g \exp 2 \varphi .
$$

If $g \in \mathscr{C}$, then $\mathscr{C}$ can be denoted by $[g]$. We assume that the metric has indefinite signature, since otherwise the compatibility condition (see below) is empty.

Two symmetric linear connections $\Gamma=\left(\Gamma_{j k}^{i}\right)$ and $\Gamma^{\prime}=\left(\Gamma_{j k}^{i}\right)$ are said to be projectively equivalent if their geodesics coincide. Here and below we consider geodesics without preferred parameterization (in literature they are sometimes called autopar-

\footnotetext{
2 More precisely EPS speak of a "Weyl space" if a light cone compatible pair of projective and conformal structures is given. In the rest of the paper they suggest that a "Weyl space" can be endowed with a Weylian metric.

3 For more details see [17].

4 "A fully rigorous formalization has not yet been achieved, but we nevertheless hope that the main line of reasoning will be intelligible and convincing to the sympathetic reader" [7, p. 69f.].

5 This has been made clear in [14, rem. 2.2] and in the preprint version of [18].
} 
allel curves). Projective equivalence is clearly an equivalence relation on the set of all symmetric linear connections on $M$. An equivalence class $\mathscr{P}$ with respect to this relation is called a projective structure; it is denoted by $[\Gamma]$ if it contains $\Gamma$. It can be formulated as the condition

$$
\Gamma \sim \Gamma^{\prime} \in \mathscr{P} \Longleftrightarrow \text { there is a } 1 \text {-form } \psi \text { so that } \Gamma_{j k}^{\prime i}=\Gamma_{j k}^{i}+\delta_{j}^{i} \psi_{k}+\delta_{k}^{i} \psi_{j}
$$

We consider here a question of Weyl's generalization of Riemannian geometry proposed in $[20,21,23]$. In the more recent literature this type of generalization has been formulated for various differential geometric structures $[10,11,16],{ }^{6}$ We use it in the sense of semi-Riemannian Weyl structures (Definition 1(i)) which are close to the Weylian manifolds (Definition 1(ii)) considered by Weyl himself.

Definition 1 (i) A (semi-Riemannian) WEYL STRUCTURE is given by triple $(M, \mathscr{C}, \nabla)$ where $M$ is a differentiable manifold, $\mathscr{C}=[g]$ a conformal class of (semi-) Riemannian metrics $g$ on $M$, and $\nabla=\nabla(\Gamma)$ the covariant derivative of a torsion free affine connection $\Gamma$, constrained by the compatibility condition that for any $g \in \mathscr{C}$ there is a differential 1-form $\varphi_{g}$ s.th. $\nabla g+2 \varphi_{g} \otimes g=0$.

(ii) A Weylian MANifold $(M,[(g, \varphi)])$ is a differentiable manifold $M$ endowed with a WEYLIAN METRIC defined by an equivalence class of pairs $(g, \varphi)$, where $g$ is a (semi-) Riemannian metric and $\varphi$ a (real valued) differential 1-form on $M$. Equivalence is defined by conformal rescaling $g \mapsto \tilde{g}=\Omega^{2} g$ and the corresponding gauge transformation for $\varphi \mapsto \widetilde{\varphi}=\varphi-d \ln \Omega$.

Weyl showed that any Weylian metric has a uniquely determined compatible affine (i.e. symmetric linear) connection $\Gamma(g, \varphi)$,

$$
\Gamma(g, \varphi)_{j k}^{i}=\digamma_{j k}^{i}+\delta_{j}^{i} \varphi_{k}+\delta_{k}^{i} \varphi_{j}-g_{j k} \varphi^{i},
$$

where $\digamma$ denotes the Levi-Civita connection of $g$ and we raised the index of $\phi_{i}$ by $g$. It is independent of the representative $(g, \varphi)$ of the Weylian metric [21]. Metric compatibility in the sense of Weyl geometry means that the the lengths of vectors parallel transported by $\Gamma(g, \varphi)$ and measured in $g$ change infinitesimally with $\varphi$. In streamlined form this means that for the covariant derivative $\nabla=\nabla(g, \varphi)$ defined by $\Gamma(g, \varphi)$ the following holds:

$$
\nabla g+2 \varphi g=0
$$

Taking into account the gauge transformation for $\varphi$ the definitions (i) and (ii) above turn out to be equivalent.

The compatibility of a projective structure with a conformal structure can now be considered from different perspectives. We use the following terminology:

Definition 2 We say that a projective structure $\mathscr{P}=[\Gamma]$ and a conformal structure $\mathscr{C}=[g]$ are

\footnotetext{
${ }^{6}$ For a concept of Weyl structures in the context of Cartan geometries modeled after a pair $(G ; P)$ with $P$ a parbolic subroup of the Lie group $G$ see [6, chap. 5].
} 
(i) LIGHT CONE COMPATIBLE if any light-like geodesic of $g \in \mathscr{C}$ is an auto-parallel for some and hence for all $\Gamma \in \mathscr{P}$;

(ii) RIEMANN COMPATIBLE if there is $g \in \mathscr{C}$ such that its Levi-Civita connection $\digamma(g) \in \mathscr{P}$

(iii) Weyl COMPATIBLE if for some $g \in \mathscr{C}$ a differential 1-form $\varphi$ can be found such that the affine connection $\Gamma(g, \varphi)$ of the Weylian metric $[(g, \varphi)]$ satisfies $\Gamma(g, \varphi) \in \mathscr{P}^{7}$

Remark 1 (i) Is independent of the choice of the connection from the projective class of $\Gamma$ and of the choice of the metric from the conformal class of $g$; it is used in [7]. We also use the abbreviation compatibility without further specification for (i). In the context of Riemannian geometry (ii) appears most natural; it is used also in [12]. (iii) Is a straight forward generalization of Riemann compatibility to the context of Weyl geometry and was implicitly considered by Weyl in [22]. A modern study of (iii) is given in [14, sec. 2.4] and [13] for the two-dimensional case; here it is called compatibility sans phrase (of a conformal and a projective structure).

Weyl compatibility implies (light cone) compatibility [21], similarly so for Riemann compatibility. On the other hand, light cone compatibility does not imply Riemann compatibility. The question remains whether light cone compatibility is strong enough to imply Weyl compatibility (assuming that the metric has indefinite signature so the light cone exists).

A trivial example of (light cone) compatible projective and conformal structures is as follows: take any two 1 -forms $\varphi=\varphi_{i}$ and $\eta=\eta_{i}$, denote by $\digamma_{j k}^{i}$ the Levi-Civita connection of any $g \in \mathscr{C}$ and consider $\mathscr{P}=[\Gamma]$ with

$$
\Gamma_{j k}^{i}=\digamma_{j k}^{i}+\eta_{j} \delta_{k}^{i}+\eta_{k} \delta_{j}^{i}-\varphi^{i} g_{j k},
$$

where we again used $g$ to raise the index of $\varphi$. Connections $\Gamma$ and $\digamma$ are obviously projectively equivalent on the light cones of $\mathscr{C}$; and this property does not depend on the choice of the representative of $\mathscr{P}$.

In our paper we prove that any pair $(\mathscr{P}, \mathscr{C})$ of compatible projective and conformal structures are related by the formula (3), see Theorem 1 . This implies that compatible $\mathscr{P}$ and $\mathscr{C}$ are also Weyl compatible, because (1) shows that in this case also the invariant affine connection of the Weylian metric $[(g, \varphi)]$ is projectively equivalent to $\Gamma$.

\section{Proof of the EPS conjecture}

Theorem 1 Let $g$ be a metric of indefinite signature on $\mathbb{R}^{n}$ with $n \geq 3$. If $\left[\Gamma_{j k}^{i}\right]$ is compatible with $[\mathrm{g}]$, then (3) holds.

Proof For any light-like geodesic $\gamma$ we have

$$
\nabla_{\dot{\gamma}}^{g} \dot{\gamma}=0 \text { and } \nabla_{\dot{\gamma}}^{\Gamma} \dot{\gamma}=\beta(\gamma, \dot{\gamma}) \dot{\gamma}
$$

\footnotetext{
${ }^{7}$ If this holds for some $g \in \mathscr{C}$, then for any $g \in \mathscr{C}$.
} 
(for some function $\beta$ ). Subtracting one equation from the other, we obtain

$$
D_{j k}^{i} \dot{\gamma}^{j} \dot{\gamma}^{k}=\beta(\gamma, \dot{\gamma}) \dot{\gamma}^{i}
$$

where the "difference" $D$ is given by $D_{j k}^{s}=\Gamma_{j k}^{s}-\digamma_{j k}^{s}$; it is a tensor.

We view (4) as a system of linear equations on the components of $D$ (assumed to be symmetric in the lower indices); the system contains infinitely many equations since as $\dot{\gamma}$ we can take any light-like vector. Our goal is to show that the general solution of this system is the one coming from (3).

For any vector $v=v^{i}$ we consider

$$
D_{j k}^{i} v^{j} v^{k} v^{s}-D_{j k}^{s} v^{j} v^{k} v^{i}
$$

This polynomial in $v$ of degree 3 expression vanishes for any $v$ such that $g(v, v)=0$; since the set $v \in \mathbb{R}^{n}$ such that $g(v, v)=0$ is an irreducible quadric, the expression (5) is divisible by $g(v, v)$, so it is equal to

$$
g(v, v) \omega_{p}^{i s} v^{p}
$$

for some $\omega_{p}^{i s}$ skew-symmetric with respect to $i, s$. On the other side, (5) has the following property: for any two 1 -forms $\sigma_{i}, \zeta_{i}$ such that $\sigma_{i} v^{i}=\zeta_{i} v^{i}=0$, if we contract (5) with $\sigma_{i} \zeta_{s}$ we obtain zero. Thus, at any $v$ and for any such $\sigma$ and $\zeta$ we have

$$
\sigma_{i} \zeta_{s} \omega_{p}^{i s} v^{p}=0
$$

Then, for any point $v$ the contravariant 2-form $\omega^{i s} v^{p}$ has rank two so it is given by $\varphi^{s} v^{i}-v^{s} \varphi^{i}$ for some $\varphi$. Let us show that this implies that

$$
D_{j k}^{i}=\delta_{j}^{i} \eta_{k}+\delta_{k}^{i} \eta_{j}-\varphi^{i} g_{j k}
$$

as we want.

In order to do this, we consider the equation

$$
D_{j k}^{i} v^{j} v^{k} v^{s}-D_{j k}^{s} v^{j} v^{k} v^{i}=\left(\varphi^{s} v^{i}-v^{s} \varphi^{i}\right) g_{j k} v^{j} v^{k}
$$

We view it as an equation on $D$ (and assume $\varphi$ is known). It should be fulfilled for all vectors $v$. It is a system of linear inhomogeneous equations. The corresponding homogeneous system is $D_{j k}^{i} v^{j} v^{k} v^{s}-D_{j k}^{s} v^{j} v^{k} v^{i}=0$. It is equivalent to the condition that for every $v$ we have that $D_{j k}^{i} v^{j} v^{k}$ is proportional to $v^{i}$. Then, its solution space is the space of pure trace tensors (i.e., of the form $D_{j k}^{i}=\delta_{j}^{i} \eta_{k}+\delta_{k}^{i} \eta_{j}$ ). Now, the general solution of an inhomogeneous system is one solution plus all solutions of the corresponding homogeneous system; since $D_{j k}^{i}=-\varphi^{i} g_{j k}$ is a solution of the system (8), the general solution is (7) as we claimed. 
Remark 2 Arguing as in [12, §2], one can extract from $D_{j k}^{i}:=\Gamma_{j k}^{i}-\digamma_{j k}^{i}=\eta_{j} \delta_{k}^{i}+$ $\eta_{k} \delta_{j}^{i}-\varphi^{i} g_{j k}$ a formula for $\varphi^{i}$ :

$$
\left(\frac{2}{n+1}-n\right) \varphi^{i}=\left(D_{j k}^{i}-\frac{1}{n+1} D_{s k}^{s} \delta_{j}^{i}-\frac{1}{n+1} D_{s j}^{s} \delta_{k}^{i}\right) g^{j k} .
$$

The Weyl structure corresponding to this $\varphi^{i}$ is integrable if the 1-form $\varphi_{i}=\varphi^{s} g_{s i}$ is closed.

Remark 3 An alternative equivalent way to solve the system (4) is as follows: the system is invariant with respect to the natural action of the group $O(g)$. Then, the solution space is also invariant and so is the direct sum of irreducible subspaces. Considering all irreducible subspaces and substituting them as ansatz in (4) (which is a standard exercise) shows that only the subspaces of tensors of the form $\eta_{j} \delta_{k}^{i}+\eta_{k} \delta_{j}^{i}$ and of the form $\varphi^{i} g_{j k}$ are solution spaces for the system.

Our proof is more elementary and works in all dimensions and all signatures (recall that decomposition in irreducible subspaces w.r.t. to the action of $O(g)$ depends on the dimension).

\section{Discussion}

Theorem 1 and discussion at the end of Sect. 2 show that EPS were right in assuming that the light cone compatibility of $(\mathscr{P}, \mathscr{C})$ is equivalent to the existence of a Weylian metric with the given projective and conformal structures. The Weylian metric is, moreover, well determined because of Weyl's uniqeness theorem, see Sect. 1. In this sense the central claim of EPS has been vindicated. ${ }^{8}$ In principle, a Weylian metric on spacetime can thus be read off from sufficiently detailed knowledge of the free fall trajectories of test particles and of the gravitational bending of light. This fact may give support for a modified gravity approach to the problem of dark matter at the astrophysical level (galaxies and galaxy clusters) $[9,18]$. The central physical question for such an approach is then whether a theoretical coherent and empirically confirmed dynamics of the underlying field content can be found.

Acknowledgements Open Access funding provided by Projekt DEAL. We thank Sergei Agafonov, Thomas Mettler, Andrzej Trautman for useful comments, and Lorenzo Fatibene for a detailed discussion. The work was started during the conference "New methods in differential geometry" supported by the DAAD (via Ostpartnerschaft programme) and by the University of Jena. V. Matveev thanks the DFG for partial support via projects MA 2565/4 and MA 2565/6.

Open Access This article is licensed under a Creative Commons Attribution 4.0 International License, which permits use, sharing, adaptation, distribution and reproduction in any medium or format, as long as you give appropriate credit to the original author(s) and the source, provide a link to the Creative Commons licence, and indicate if changes were made. The images or other third party material in this article are included in the article's Creative Commons licence, unless indicated otherwise in a credit line to the material. If material is not included in the article's Creative Commons licence and your intended use is not permitted

\footnotetext{
8 We do not deal with those parts of the paper in which the differentiable structure on $M$ and the structures
} $\mathscr{P}, \mathscr{C}$ are derived from the rather involved "axioms" of [7]. 
by statutory regulation or exceeds the permitted use, you will need to obtain permission directly from the copyright holder. To view a copy of this licence, visit http://creativecommons.org/licenses/by/4.0/.

\section{References}

1. Audretsch, J.: Riemannian structure of space-time as a consequence of quantum mechanics. Phys. Rev. D 27, 2872-2884 (1983)

2. Audretsch, J., Gähler, F., Norbert, S.: Wave fields in Weyl spaces and conditions for the existence of a preferred pseudo-riemannian structure. Commun. Math. Phys. 95, 41-51 (1984)

3. Audretsch, J., Hehl, F.W., Lämmerzahl, C.: Matter wave interferometry and why quantum objects are fundamental for establishing a gravitational theory. In: Ehlers, J., Schaefer, G. (eds.) Relativistic Gravity Research with Emphasis on Experiments and Observations. Lecture Notes in Physics, vol. 410, pp. 369-407. Springer, Berlin (1992)

4. Audretsch, J., Lämmerzahl, C.: A new constructive axiomatic scheme for the geometry of space-time. In: Majer, U., Schmidt, H.-J. (eds.) Semantical Aspects of Spacetime Theories, pp. 21-40. BI-Verlag, Mannheim (1994)

5. Coleman, R., Korté, H.: Constraints on the nature of inertial motion arising from the universality of free fall and the conformal causal structure of spacetime. J. Math. Phys. 25, 3513-3526 (1984)

6. Căp, A., Slovák, J.: Parabolic Geometries I. Background and General Theory. Mathematical Surveys and Monographs, vol. 154. American Mathematical Society, Providence (2009)

7. Ehlers, J., Pirani, F., Schild, A.: The geometry of free fall and light propagation. In: O'Raifertaigh, L. (ed.) General Relativity, Papers in Honour of J.L. Synge, pp. 63-84. Clarendon Press, Oxford (1972). Republished in General Relativity and Gravity (2012), 44:1587-1609

8. Einstein, A.: The Collected Papers of Albert Einstein. University Press, Princeton (1987)

9. Fatibene, L., Francaviglia, M.: Extended theories of gravitation and the curvature of the universeDo we really need dark matter? In: Olmo, Gonzalo J. (ed.) Open Questions in Cosmology. InTechOpen, London (2012). chapter 5. https://www.intechopen.com/books/open-questions-incosmology/extended-theories-of-gravitation-and-the-curvature-of-the-universe-do-we-really-needdark-matter-

10. Gilkey, P., Nikcevic, S., Udo, S.: Geometric realizations, curvature decompositions, and Weyl manifolds. J. Geom. Phys. 61, 270-275 (2011). arXiv:1002.5027

11. Higa, T.: Weyl manifolds and Einstein-Weyl manifolds. Comment. Math. Sancti Pauli 42, 143-160 (1993)

12. Matveev, V.S., Trautman, A.: A criterion for compatibility of conformal and proojective structures. Commun. Math. Phys. 329, 821-825 (2014)

13. Mettler, T.: Weyl metrisability of two-dimensional projective structures. Math. Proc. Camb. Philos. Soc. 156, 99-113 (2014)

14. Mettler, T.: Extremal conformal structures on projective surfaces. To appear in Annali della Scuola Normale Superiore Pisa - Classe di Scienze. (2015). arXiv:1510.01043

15. O'Raifeartaigh, L.: The Dawning of Gauge Theory. University Press, Princeton (1997)

16. Ornea, L.: Weyl structures in quaternionic geomety. A state of the art. In: Barletta, E. (ed.) Selected Topics in Geometry and Mathematical Physics, vol. 1, pp. 43-80. Univ. degli Studi della Basilicata, Potenza (2001). arXiv:math/0105041

17. Scholz, E.: The unexpected resurgence of Weyl gometry in late 20-th century physics. In: Walter, S., Rowe, D., Sauer, T. (eds.) Beyond Einstein. Perspectives on Geometry, Gravitation and Cosmology, Einstein Studies, vol. 13, pp. 261-360. Birkhäuser-Springer, Basel (2018). arXiv:1703.03187

18. Scholz, E.: A scalar field inducing a non-metrical contribution to gravitational acceleration and a compatible add-on to light deflection (2019). Preprint. arxiv:1906.04989

19. Trautman, A.: Editorial note to J. Ehlers, F.A.E. Pirani and A. Schild, The geometry of free fall and light propagation. Gen. Relativ. Gravit. 44, 1581-1586 (2012)

20. Weyl, H.: Gravitation und Elektrizität. Sitzungsberichte der Königlich Preußischen Akademie der Wissenschaften zu Berlin, pp. 465-480. In [25, II, 29-42], English in [15, 24-37] (1918)

21. Weyl, H.: Reine Infinitesimalgeometrie. Math. Z. 2, 384-411 (1918b). In [25, II, 1-28]

22. Weyl, H.: Zur Infinitesimalgeometrie: Einordnung der projektiven und der konformen Auffassung. Nachrichten Göttinger Gesellschaft der Wissenschaften, pp. 99-112 (1921). In [25, II, 195-207] 
23. Weyl, H.: Space-Time-Matter. Translated from the 4th German edition by H. Brose. Methuen, London (1922). Reprint New York: Dover 1952

24. Weyl, H.: Mathematische Analyse des Raumproblems. Vorlesungen gehalten in Barcelona und Madrid. Springer, Berlin etc. Nachdruck Darmstadt: Wissenschaftliche Buchgesellschaft 1963 (1923)

25. Weyl, H.: In: Chandrasekharan, K. (ed.) Gesammelte Abhandlungen, vol. 4. Springer, Berlin (1968)

Publisher's Note Springer Nature remains neutral with regard to jurisdictional claims in published maps and institutional affiliations. 